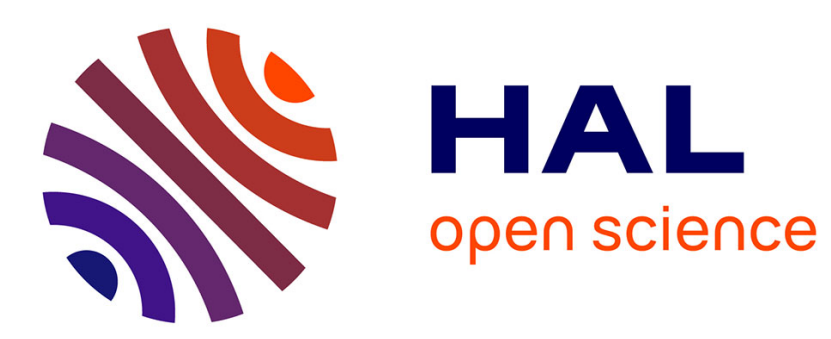

\title{
Heterogeneous reaction induced by the piston effect in supercritical binary mixtures
}

Isabelle Raspo, Sofiane Meradji, Bernard Zappoli

\section{To cite this version:}

Isabelle Raspo, Sofiane Meradji, Bernard Zappoli. Heterogeneous reaction induced by the piston effect in supercritical binary mixtures. Chemical Engineering Science, 2007, 62 (16), pp.4182-4192. 10.1016/j.ces.2007.04.027 . hal-01139223

\section{HAL Id: hal-01139223 \\ https://hal.science/hal-01139223}

Submitted on 3 Apr 2015

HAL is a multi-disciplinary open access archive for the deposit and dissemination of scientific research documents, whether they are published or not. The documents may come from teaching and research institutions in France or abroad, or from public or private research centers.
L'archive ouverte pluridisciplinaire HAL, est destinée au dépôt et à la diffusion de documents scientifiques de niveau recherche, publiés ou non, émanant des établissements d'enseignement et de recherche français ou étrangers, des laboratoires publics ou privés. 


\title{
Heterogeneous Reaction induced by the Piston Effect in Supercritical Binary Mixtures
}

\author{
Isabelle RASPO ${ }^{\mathrm{a},{ }^{*}}$, Sofiane MERADJI ${ }^{\mathrm{a}}$, Bernard ZAPPOLI ${ }^{\mathrm{b}}$ \\ a MSNM-GP, UMR 6181 CNRS, Paul-Cezanne University, IMT-La Jetée, Technopôle de Château- \\ Gombert, 38 rue Frédéric Joliot Curie, 13451 Marseille Cedex 20, France \\ ${ }^{\mathrm{b}}$ CNES, 18 avenue Edouard Belin, 31401 Toulouse Cedex 4, France
}

\begin{abstract}
It is now well established that the large compressibility of supercritical fluids is responsible for the strong enhancement of the thermo-acoustic heating, leading to the speeding up of the heat transport thanks to the piston effect instead of the expected slowing down. We show in this paper, through numerical simulations, that the hydrodynamics behavior of supercritical fluids also couples with the critical behavior of the solubility of solids to cause the release of a heterogeneous reaction at solid surfaces in dilute binary supercritical mixtures.
\end{abstract}

Keywords: supercritical fluids, multiphase reactions, hydrodynamics, solubility, piston effect, numerical analysis

\footnotetext{
* Corresponding author.

Email address : $\underline{\text { isabel@L3m.univ-mrs.fr }}$
}

Tel: 33 (0) 491118546

Fax: 33 (0) 491118502 


\section{Introduction}

A supercritical fluid is any substance set above the temperature and pressure of its gas-liquid critical point. In this quite special state, the physical properties of the fluid are intermediate between those of liquids and gases. It exhibits a liquid-like density but mass transport properties, such as viscosity, are close to those of gases improving its mass transport rate. Moreover, as the critical point is approached, the isothermal compressibility and the thermal expansion coefficient diverge, while the thermal diffusivity tends to zero. As a consequence, density changes significantly for minor variations of pressure or temperature. More precisely, if we note $\varepsilon=\left(T-T_{c}\right) / T_{c}$ (with $T_{c}$ the critical temperature) the dimensionless distance to the critical point on the critical isochore, relative density perturbations are $\varepsilon^{-1.24}$ times larger than the temperature ones in thermal diffusion layers, especially near isothermal boundaries (Zappoli et al., 1990). The strong compressibility and dilatability of supercritical fluids induce several specific behaviors, in particular a very fast heat transport, due to thermo-acoustic effects (known as Piston Effect), whereas heat diffusion is very slow. Indeed, the heating of a confined supercritical fluid provokes a very thin diffusion boundary layer which strongly expands and compresses adiabatically the rest of fluid. This process results in a very fast and homogeneous increase of the temperature and pressure in the whole fluid. The piston effect was theoretically predicted for a pure supercritical fluid in the early nineties by several teams (Boukari et al., 1990, Onuki et al., 1990, Zappoli et al., 1990), and then it was extensively studied in microgravity and normal gravity conditions (Garrabos et al., 1998, Guenoun et al., 1993, Kogan and Meyer, 1998, Straub et al., 1995, Zhong et al., 1997). Since the strong compressibility of supercritical fluids deeply 
changes the leading mechanism of heat transfer, it was most likely that the induced pressure and density variations might also have consequences on the behavior of an interface reaction, and associated mass transfers, in supercritical mixtures and our aim was to check this assumption. More precisely, we were interested in the case of an adsorption-desorption reaction taking place between a solid interface and an active compound in a dilute binary supercritical mixture. Now, the solubility of solids in supercritical fluids is very sensitive to small changes of temperature and pressure near the solvent's critical point. Indeed, the phase diagram of binary mixtures of interest to explore this kind of heterogeneous reaction in supercritical mixtures exhibits a crossing point (named the Lower Critical EndPoint (LCEP)) between the solid-liquid-vapor coexistence line and the critical line of the mixture starting at the solvent's critical point (Fig. 1). Above this crossing point and up to the upper critical endpoint (UCEP), there exists a phase equilibrium, in the whole range of pressure and composition, between a pure solid phase of one of the compounds and the supercritical mixture. At the LCEP, the solubility of the solid changes with an infinite slope as a function of temperature on the isobar and as a function of pressure on the isotherm. So, just above this critical endpoint, small changes of temperature or pressure cause large variations of the solubility, and the LCEP temperature is usually a few Kelvin above the critical temperature of the solvent. Therefore, in the vicinity of the LCEP, the change of pressure induced by the piston effect should influence the reaction at the solidsupercritical fluid interface because it will cause a strong change of the solubility of the solid. Moreover, the behavior of the solubility of solids in supercritical fluids is made more complex by the existence of the retrograde phenomenon, namely the decrease of the solubility with an isobaric increase of temperature for a certain range of pressures including the LCEP pressure. Since the beginning of the eighties, the solubility of solids 
in supercritical fluids was extensively studied, but these studies were essentially devoted to measurements of the solubility of various compounds and to the development of thermodynamic models allowing to obtain a good correlation of experimental data (see the review paper of Higashi et al., 2001). Several papers were also published concerning measurements or modeling of adsorption equilibrium of various solutes in supercritical fluids (Afrane and Chimowitz, 1995, Uchida et al., 1997, Tapia-Corzo et al., 2000, Jha and Madras, 2004), but these models did not take into account hydrodynamics. To the authors' knowledge, no investigation was previously performed on heterogeneous reactions in supercritical mixtures, taking into account at the same time the specific supercritical hydrodynamics and the critical behavior of the solubility of solids in supercritical fluids. A first attempt using asymptotic analysis was published by Zappoli and Carles (1996) but the very simple model, based on the van der Waals equation of state with linear mixing rules, did not take into account the critical behavior of the solubility with respect to pressure and neglected the solubility variations with temperature. In this paper, by means of numerical simulations based on the solution of the complete Navier-Stokes equations, we show that, close to the LCEP, the piston effect, coupled with the critical behavior of the solubility, can be responsible for the release of a heterogeneous reaction at a chemically active surface in a dilute binary supercritical mixture.

\section{Physical problem and modeling}

\subsection{Problem under study}

The Naphthalene- $\mathrm{CO}_{2}$ mixture was chosen as a reference system with the abovementioned phase diagram because of the wide experimental data on solubility which can 
be found in literature, allowing an easier validation of our thermodynamic model (Nicolas et al., 2005). The physical properties of each pure compound are given in table 1. We consider a dilute mixture of Naphthalene (the minority species, named species 2) and supercritical $\mathrm{CO}_{2}$ (named species 1), confined between two infinite solid plates that are spaced by a distance $L=1 \mathrm{~cm}$ and made of pure solid Naphthalene (Fig. 2). Naphthalene can be adsorbed or desorbed at the two plates following a diffusion-limited reaction. The fluid is initially at rest, in thermodynamic equilibrium at a uniform temperature $T_{i}$, slightly above the LCEP temperature $T_{L C E P}=307.65 \mathrm{~K}$ (Tsekhanskaya et al., 1962), $\left(T_{i}=(1+\varepsilon) T_{L C E P}\right.$ with $\varepsilon=\left(T_{i}-T_{L C E P}\right) / T_{L C E P}<<1$ the dimensionless distance to the LCEP on the isochore $\rho=\rho_{\text {LCEP }}$ ), at the LCEP density $\rho_{L C E P}=470 \mathrm{~kg} \cdot \mathrm{m}^{-3}$ (which is not very different from the critical density of $\mathrm{CO}_{2}$ ) and in equilibrium with the solid plates. We note that $T_{L C E P}$ is only a few Kelvin above the critical temperature of $\mathrm{CO}_{2}$. Then, the temperature of the solid plate at $x=0$ is gradually increased up to $T_{i}+\Delta T$ (with $\Delta T$ about some $\mathrm{mK}$ ) whereas the other one (at $x=L)$ is kept at the initial temperature. In order to suppress the buoyant convective transport, we assume that the system is under zero gravity conditions. In such a case, the system essentially evolves in the direction of the temperature gradient and a one-dimensional model can be rightfully considered.

\subsection{Governing equations}

The evolution of the system is governed by the one-dimensional time-dependent and compressible Navier-Stokes equations, coupled with energy and mass diffusion equations. An equation of state completes the model. Since we are interested in phenomena occurring at the time scale of the piston effect which is much longer than the acoustic one, the Low Mach number approximation (Paolucci, 1982) is used to filter 
acoustic waves, allowing to strongly reduce computational costs. This approximation leads to a splitting of the pressure $P$ into a homogeneous thermodynamic part $P_{t h}(t)$, appearing in the equation of state and in the energy equation, and a non-homogeneous dynamic part $p(x, t)$, appearing in the momentum equation. For the problem treated here, the dynamic pressure is extremely small compared to the thermodynamic part (about $10^{5}$ times smaller). As a consequence, the total pressure $P$ is almost equal to the thermodynamic pressure $P_{t h}$. This latter is computed through the global mass conservation. The governing equations are thus:

$$
\begin{aligned}
& \frac{\partial \rho}{\partial t}+\frac{\partial}{\partial x}(\rho u)=0 \\
& \frac{\partial}{\partial t}(\rho u)+\frac{\partial}{\partial x}(\rho u u)=-\frac{\partial p}{\partial x}+\frac{\partial}{\partial x}\left(\eta \frac{\partial u}{\partial x}\right) \\
& \frac{\partial}{\partial t}(\rho w)+\frac{\partial}{\partial x}(\rho u w)=\frac{\partial}{\partial x}\left(\rho D_{21} \frac{\partial w}{\partial x}\right) \\
& \frac{\partial}{\partial t}(\rho \Lambda T)+\frac{\partial}{\partial x}(\rho u \Lambda T)=-P_{t h} \frac{\partial u}{\partial x}+\frac{\partial}{\partial x}\left(\lambda \frac{\partial T}{\partial x}\right)-\Gamma \frac{\partial}{\partial x}\left(\rho D_{21} \frac{\partial w}{\partial x}\right)
\end{aligned}
$$

where $u$ is the velocity, $w$ is the mass fraction of Naphthalene, $\eta$ is the dynamic viscosity, $\lambda$ is the thermal conductivity, $D_{21}$ is the diffusion coefficient of Naphthalene in supercritical $\mathrm{CO}_{2}$, and $\Lambda$ and $\Gamma$ are respectively a function of $(T, \rho, w)$ and a constant that are given in appendix A. We supposed that the transport properties of the mixture are those of pure $\mathrm{CO}_{2}$, which is a valid assumption for a dilute mixture $(w<<1)$. The following correlations are then used for the thermal conductivity $\lambda\left(\mathrm{W} \cdot \mathrm{m}^{-1} \cdot \mathrm{K}^{-1}\right)$ and the dynamic viscosity $\eta$ (Pa.s) of $\mathrm{CO}_{2}$ (Arai et al., 2002):

$$
\begin{aligned}
& \lambda(T, \rho)=\lambda_{0}(T)+\lambda_{e}(\rho)+\Delta \lambda_{c}(T, \rho), \\
& \eta(\rho)=\eta_{0}+\eta_{e}(\rho),
\end{aligned}
$$


where the first term corresponds to the limit of small densities:

$\lambda_{0}(T)=-7.6683 \times 10^{-3}+8.0321 \times 10^{-5} T$ and $\eta_{0}=1.5305 \times 10^{-5}$,

the second term is the excess property:

$\lambda_{e}(\rho)=3.0990 \times 10^{-5} \rho+5.5782 \times 10^{-8} \rho^{2}+2.5990 \times 10^{-17} \rho^{5}$,

$\eta_{e}(\rho)=5.5934 \times 10^{-9} \rho+6.1757 \times 10^{-11} \rho^{2}+2.6430 \times 10^{-17} \rho^{4}$,

and the third term is the critical enhancement, which is neglected here for the viscosity:

$\Delta \lambda_{c}(T, \rho)=\left(\frac{1.6735}{T-291.4686}-0.2774+7.4216 \times 10^{-4} T\right) \exp \left(-C^{2}\left(\rho-\rho_{c 1}\right)^{2}\right)$

with: $\left\{\begin{array}{ll}C=6.7112 \times 10^{-3} & \text { if } \rho<\rho_{c 1} \\ C=6.9818 \times 10^{-3} & \text { if } \rho>\rho_{c 1}\end{array}\right.$.

The diffusion coefficient of Naphthalene in supercritical $\mathrm{CO}_{2}, D_{21}\left(\mathrm{~m}^{2} . \mathrm{s}^{-1}\right)$, is calculated with the Wilke-Chang equation (Higashi et al., 2001):

$D_{21}=7.4 \times 10^{-15} \frac{T \sqrt{\phi M_{1}}}{\eta v_{b 2}^{0.6}}$

with $v_{b 2}\left(\mathrm{~cm}^{3} \cdot \mathrm{mol}^{-1}\right)$ the molar volume of Naphthalene at boiling point, $\phi$ the association factor $\left(\phi=1\right.$ for $\mathrm{CO}_{2}$ ) and $M_{1}$ (expressed here in $\mathrm{g} \cdot \mathrm{mol}^{-1}$ ) the molecular weight of $\mathrm{CO}_{2}$.

The thermodynamic state of the mixture is described by the Peng-Robinson equation of state in the framework of the one-fluid theory:

$P_{t h}=\frac{\left(R / M_{1}\right) T \rho \theta(w)}{1-b(w) \rho / \theta(w)}-\frac{a(T, w) \rho^{2}}{1+2 b(w) \rho / \theta(w)-b(w)^{2} \rho^{2} / \theta(w)^{2}}$,

where $R$ is the perfect gas constant $\left(R=8.3145 \mathrm{~J} \cdot \mathrm{mol}^{-1} \cdot \mathrm{K}^{-1}\right)$ and: 


$$
\begin{aligned}
& \theta(w)=1-\left(1-M_{1} / M_{2}\right) w, \quad a(T, w)=a_{1}(T)(1-w)^{2}+2 a_{12}(T) w(1-w)+a_{2}(T) w^{2}, \\
& b(w)=b_{1}(1-w)^{2}+2 b_{12} w(1-w)+b_{2}\left(M_{1} / M_{2}\right) w^{2}, \\
& a_{j}(T)=1.48742 \frac{R}{M_{j}} \frac{T_{c j}}{\rho_{c j}}\left[1+\beta_{j}\left(1-\sqrt{\frac{T}{T_{c j}}}\right)\right]^{2}, b_{j}=0.25307 \frac{1}{\rho_{c j}}, \\
& \beta_{j}=0.37464+1.54226 \omega_{j}-0.26992 \omega_{j}^{2}, \quad(j=1,2), \\
& a_{12}(T)=\sqrt{a_{1}(T) a_{2}(T)}\left(1-k_{12}\right), b_{12}=\frac{1}{2}\left(b_{1} \frac{M_{1}}{M_{2}}+b_{2}\right)\left(1-l_{12}\right),
\end{aligned}
$$

with $M_{2}\left(\mathrm{~kg} \cdot \mathrm{mol}^{-1}\right)$ the molecular weight of Naphthalene and $\omega$ the acentric factor. The binary interaction parameters $k_{12}$ and $l_{12}$ are determined so as to minimize the error between the solubility (or equilibrium mole fraction $y_{2}^{e}$ ) calculated by the model and the experimental data, using a least squares method. More precisely, the minimized objective function is:

$$
F^{o b j}=\sum_{i=1}^{N}\left[\frac{\left(y_{2}^{e}\right)_{i}^{\exp }-\left(y_{2}^{e}\right)_{i}^{c a l}}{\left(y_{2}^{e}\right)_{i}^{\exp }}\right]^{2},
$$

where $N$ is the number of experimental values and $\left(y_{2}^{e}\right)_{i}^{\exp }$ and $\left(y_{2}^{e}\right)_{i}^{c a l}$ are respectively the experimental values and those estimated by the model. Nicolas et al. (2005) have shown that temperature dependent binary parameters are necessary to obtain a reliable correlation of solubility data in a wide range of temperatures. Therefore, the following formula was considered for $k_{12}$ and $l_{12}$ :

$$
\begin{aligned}
& k_{12}=k_{12}^{\prime}+k_{12}^{\prime \prime}\left(\frac{308.15}{T}-1\right) \\
& l_{12}=l_{12}^{\prime}+l_{12}^{\prime \prime}\left(\frac{308.15}{T}-1\right)
\end{aligned}
$$


The model consists in determining the solubility $y_{2}^{e}$ of Naphthalene in supercritical $\mathrm{CO}_{2}$ from the equilibrium condition, namely the equality of Naphthalene fugacity in the solid and supercritical phases. This equilibrium condition leads to the following equation for the solubility:

$$
y_{2}^{e}=\frac{\varphi_{2}\left(T, P_{2}^{s u b}\right) P_{2}^{s u b}(T)}{P \varphi_{2}\left(T, P, y_{2}^{e}\right)} \exp \left(\frac{v_{2}^{s}\left(P-P_{2}^{s u b}(T)\right)}{R T}\right)
$$

where $P_{2}^{\text {sub }}(T)$ (computed by the Antoine equation) is the sublimation pressure of Naphthalene and $v_{2}{ }^{s}$ its molar volume at temperature $T$; it is assumed that $v_{2}^{s}$ is independent on pressure $P$. The fugacity coefficients $\varphi_{2}\left(T, P_{2}^{s u b}\right)$ and $\varphi_{2}\left(T, P, y_{2}^{e}\right)$ are respectively those of Naphthalene as a pure species at $P_{2}{ }^{\text {sub }}(T)$ and in the supercritical mixture at pressure $P$; they are estimated using the equation of state (6) written for the total pressure $P$ (instead of $P_{t h}$ ) and for the molar variables, $y_{2}$ and $v$, which are linked to specific variables by the formula:

$$
y_{2}=\frac{M_{1}}{M_{2}} \frac{w}{\theta(w)}, v=\frac{M_{1}}{\theta(w) \rho}
$$

In fact, since the fugacity coefficient $\varphi_{2}\left(T, P_{2}^{s u b}\right)$ differs little from 1 , it was approximated by 1 in Eq. (9). A mean relative deviation of $8.4 \%$ between experimental values and those calculated with Eq. (9) was obtained in the temperature range $[308.15 \mathrm{~K}, 328.15 \mathrm{~K}]$ in which solubility data are available. The values of the binary interaction parameters are then: $\quad k^{\prime}{ }_{12}=0.0395, \quad k{ }_{12}=0.0114, \quad l_{12}^{\prime}=-0.1136$ and $l{ }_{12}=-0.3103$. The solubility of Naphthalene, predicted by the model with these values, versus pressure is drawn for three isotherms in Fig. 3. This figure clearly shows the zone of strong variation of the solubility around the LCEP pressure. Since the heating of 
the plate is very small (some $\mathrm{mK}$ ), the binary parameters $k_{12}$ and $l_{12}$ are calculated for the initial temperature using Eq. (8) and, then, they are kept constant during the simulation.

\subsection{Solid-fluid interface reaction}

The reaction at interfaces $x=0$ and $x=L$ is supposed to be diffusion-limited. Thus, the fluid phase is assumed to be locally in equilibrium with the solid interface at any time, namely the partial density of Naphthalene is equal to its equilibrium value $\rho_{2}^{e}(T, P)$ leading to the following boundary condition for $w$ :

$$
w(x=0 \text { or } L, t)=\frac{w_{c}^{e}(T, P)}{\rho(x=0 \text { or } L, t) / \rho_{L C E P}},
$$

with $w_{c}^{e}(T, P)=\rho_{2}^{e}(T, P) / \rho_{L C E P}$ the equilibrium mass fraction for the critical density $\rho_{\text {LCEP. }}$. The equilibrium partial density of Naphthalene is computed from its solubility $y_{2}^{e}(T, P)$ by $\rho_{2}^{e}=M_{2} y_{2}^{e} / v$, with $v$ the molar volume of the mixture given by the PengRobinson equation for $T, P$, and $y_{2}^{e}(T, P)$. That is why it is necessary to accurately represent the solubility behavior. The boundary condition for the velocity is obtained through the Fick's law:

$$
u(x=0 \text { or } L, t)=-\frac{D_{21}}{w(x=0 \text { or } L, t)-1} \frac{\partial w}{\partial n}(x=0 \text { or } L, t)
$$

with $n$ the outer normal to the boundary. 


\subsection{Numerical method}

The governing equations (1)-(4) and (6) are solved in a dimensionless form using $T_{L C E P}$ as reference temperature, $\rho_{L C E P}$ as reference density, $\rho_{L C E P}\left(R / M_{1}\right) T_{L C E P}$ as reference pressure, $L$ as reference length, the time scale of the piston effect as reference time $\left(t_{P E}=\left(t_{d} / \operatorname{Pr}\right) \varepsilon^{3 / 2}\right.$, where $\operatorname{Pr}=\eta C_{p} / \lambda$ is the Prandtl number for $\mathrm{CO}_{2}$, with $C_{p}$ the specific heat at constant pressure that is calculated from the Peng-Robinson equation of state (see appendix $\mathrm{B}$ ), and $t_{d}$ is the characteristic time of thermal diffusion) and the corresponding velocity (i.e. $L / t_{P E}$ ) as reference velocity.

The numerical method, which is based on finite volume approximations, is very similar to that developed by Amiroudine et al. (1997). The time scheme is fully implicit with the power-law scheme used for the discretization of the convective terms. On the other hand, the unsteady terms are approached by a $2^{\text {nd }}$ order backward Euler scheme whereas a $1^{\text {st }}$ order scheme was used by Amiroudine et al. We used a non-uniform staggered mesh: the scalar variables are computed at the cells centre while the velocity is defined at the cells faces. The mesh, which includes 201 points, is refined near the boundaries so as to accurately represent thin boundary layers (about 20\% of grid points inside the boundary layers). The velocity-pressure coupling of the Navier-Stokes equations is treated by the SIMPLER (SIMPLE-Revised) algorithm and numerical stability in terms of time steps and grid sizes has been carefully checked. 


\section{Results and discussion}

\section{1-General description}

Simulations were performed for initial temperatures $T_{i}$ ranging from $307.75 \mathrm{~K}$ to $323.15 \mathrm{~K}$ (i.e. a dimensionless distance to the LCEP, $\varepsilon$, ranging from $3.2 \times 10^{-4}$ to $5 \times 10^{-2}$ ) and a temperature increase $\Delta T=2 \mathrm{mK}, 5 \mathrm{mK}$ and $10 \mathrm{mK}$ at the plate $x=0$. It must be noted that $T_{i}=307.75 \mathrm{~K}$ (that is only $0.1 \mathrm{~K}$ above the LCEP temperature) is out of the temperature range for which the model parameters $k_{12}, l_{12}$ were fitted on solubility data. The values of the binary parameters $k_{12}, l_{12}$ are computed from Eq. (8) but the accuracy of the model at this temperature cannot be evaluated since there is no available solubility data. The values of the initial pressure $P_{i}$ and mass fraction of Naphthalene $w_{i}$, respectively obtained through the equation of state and the equilibrium condition (9), are given in table 2 for each initial temperature. Table 2 also presents, for each initial states, the values of the thermal diffusivity $\alpha$ of $\mathrm{CO}_{2}$ calculated from the model as explained in appendix $\mathrm{B}$, of the diffusion coefficient $D_{21}$ of Naphthalene in supercritical $\mathrm{CO}_{2}$ calculated with Eq. (5), and various characteristic times, namely the thermal diffusion time $t_{d}$, the mass diffusion time $t_{M d}$ and the time scale of the piston effect $t_{P E}$. It can be noted that the diffusion coefficient $D_{21}$, as the thermal diffusivity $\alpha$, is very small (about $10^{-8}$ ) but, unlike $\alpha$, it little changes in the temperature range that we considered. In fact, some measurements revealed that the binary diffusion coefficient in supercritical fluids strongly decreases in the vicinity of the LCEP (Higashi et al., 1998, Nishiumi et al., 1996, Tsekhanskaya, 1971) but the Wilke-Chang equation does not represent this decrease. As a consequence, the characteristic time of mass diffusion $t_{M d}$ remains almost constant for all the values of $T_{i}$ and it is about $1 \mathrm{~h} 10 \mathrm{~min}$. Table 2 also shows that the time scale of the piston effect is very much smaller than the two other 
characteristic times. In particular, $t_{P E}$ is about 45 thousand times smaller than the characteristic time of mass diffusion at $T_{i}=308.15 \mathrm{~K}$ and it remains 252 times smaller than $t_{M d}$ at $T_{i}=323.15 \mathrm{~K}$, i.e. at $15.5 \mathrm{~K}$ above the LCEP.

In all the cases, the same phenomenon was observed. We discuss here the case $T_{i}=308.15 \mathrm{~K}$ and $\Delta T=10 \mathrm{mK}$. The temperature of the plate $x=0$ is gradually increased using a cosine law during $t_{\text {heat }}=10 t_{P E}$, i.e. approximately $1 \mathrm{~s}$. More precisely, the temperature increase is defined by:

$$
\delta T(t)= \begin{cases}0.5 \Delta T\left(1-\cos \left(\pi \frac{t}{t_{\text {heat }}}\right)\right) & \text { if } t \leq t_{\text {heat }} \\ \Delta T & \text { if } t>t_{\text {heat }}\end{cases}
$$

As in the case of a pure supercritical fluid, the boundary heating induces a thin thermal diffusion layer, associated with a large density gradient, near the heated plate. Due to the high dilatability of the fluid, this boundary layer strongly expands and compresses adiabatically the rest of the fluid leading to a fast and homogeneous increase of its temperature by the piston effect (Fig. 4). The fluid near the isothermal boundary is then warmer than the solid plate and a second thermal boundary layer forms near this latter where diffusion cools the fluid. The fluid layer is therefore divided into three zones: two thermal boundary layers, associated with large density gradients (Fig. 5), and the isothermal bulk of the fluid (Fig. 4). We noted that the velocity profiles are also similar to those obtained in a pure supercritical fluid. Therefore, the thermomechanical response of the mixture to the heating of the plate is the same as that of the pure supercritical fluid. 
We consider now the chemical response of the system to the heating of the plate. Since the temperature increase at $x=0$ is very small, the boundary condition (11) for $w$ can be written at first order as:

$$
\begin{aligned}
w(x=0, t) & =w_{i}+\left[\left(\frac{\partial w_{c}^{e}}{\partial T}\right)_{P}\left(T_{i}, P_{i}\right)\right]\left(T(x=0, t)-T_{i}\right) \\
& +\left[\left(\frac{\partial w_{c}^{e}}{\partial P}\right)_{T}\left(T_{i}, P_{i}\right)\right]\left(P(x=0, t)-P_{i}\right)-\frac{w_{i}}{\rho_{i}}\left(\rho(x=0, t)-\rho_{i}\right) .
\end{aligned}
$$

with:

$$
\begin{aligned}
\left(\frac{\partial w_{c}^{e}}{\partial T}\right)_{P}\left(T_{i}, P_{i}\right) & =\left(1-\left(1-\frac{M_{1}}{M_{2}}\right) w_{i}\right)\left[\frac{M_{2}}{M_{1}}-\frac{\rho_{i} w_{i}}{M_{1}} v_{2}\left(T_{i}, P_{i}\right)\right]\left(\frac{\partial y_{2}^{e}}{\partial T}\right)_{P}\left(T_{i}, P_{i}\right) \\
& -w_{i} \beta_{P, y_{2}}\left(T_{i}, P_{i}\right) \\
\left(\frac{\partial w_{c}^{e}}{\partial P}\right)_{T}\left(T_{i}, P_{i}\right) & =\left(1-\left(1-\frac{M_{1}}{M_{2}}\right) w_{i}\right)\left[\frac{M_{2}}{M_{1}}-\frac{\rho_{i} w_{i}}{M_{1}} v_{2}\left(T_{i}, P_{i}\right)\right]\left(\frac{\partial y_{2}^{e}}{\partial P}\right)_{T}\left(T_{i}, P_{i}\right) \\
& +w_{i} \chi_{T, y_{2}}\left(T_{i}, P_{i}\right)
\end{aligned}
$$

where $v_{2}\left(T_{i}, P_{i}\right)=\left(\partial v / \partial y_{2}\right)_{T, P}\left(T_{i}, P_{i}\right)$ is the partial molar volume of Naphthalene (which is negative), $\beta_{P, y_{2}}\left(T_{i}, P_{i}\right)=(\partial v / \partial T)_{P, y_{2}}\left(T_{i}, P_{i}\right) / v_{i}$ is the thermal expansion coefficient at constant composition for $y_{2}=y_{2}^{e}\left(T_{i}, P_{i}\right)$, and $\chi_{T, y_{2}}\left(T_{i}, P_{i}\right)=-(\partial v / \partial P)_{T, y_{2}}\left(T_{i}, P_{i}\right) / v_{i}$ is the isothermal compressibility at constant composition for $y_{2}=y_{2}^{e}\left(T_{i}, P_{i}\right)$.

An equation similar to Eq. (13) (without the term due to the temperature perturbation) can be written for $w(x=L, t)$. Equations (14) and (15) show that the derivatives of $w_{c}^{e}$ depends not only on the solubility derivatives (which are very large 
near the LCEP) but also on the thermal expansion coefficient and on the isothermal compressibility that are also very large since the solvent is supercritical. The contribution of these two coefficients increases the term due to the solubility derivatives, so that the derivatives of $w_{c}^{e}$ are about 4 times larger than the solubility ones. For $T_{i}=308.15 \mathrm{~K}$, we have $\left(\partial y_{2}^{e} / \partial T\right)_{P}\left(T_{i}, P_{i}\right)=-3.78 \times 10^{-3} K^{-1} \quad$ and $\left(\partial y_{2}^{e} / \partial P\right)_{T}\left(T_{i}, P_{i}\right)=2.21 \times 10^{-8} \mathrm{~Pa}^{-1}$; therefore a temperature change of $1 \mathrm{~K}$ (namely about $0.3 \%$ ) induces a solubility variation equal to $3.78 \times 10^{-3}$ (namely about $142 \%$ !) and a pressure change of 1 bar (namely about $1 \%$ ) induces a solubility variation equal to $2.21 \times 10^{-3}$ (namely about $83 \% !$ ).

Equation (13) shows that the evolution of the mass fraction of Naphthalene at the solid interfaces is governed by three mechanisms:

1. The critical behavior of the solute solubility with respect to temperature and pressure through the very large values of the derivatives $\left(\partial w_{c}^{e} / \partial T\right)_{P}$ and $\left(\partial w_{c}^{e} / \partial P\right)_{T}$, respectively in the terms due to the temperature and pressure perturbations; of course, the critical behavior of the solubility with respect to temperature acts only at the heated plate;

2. The strong compressibility of the supercritical fluid through the derivative $\left(\partial w_{c}^{e} / \partial P\right)_{T}$, and the piston effect, that results from it, through the pressure variation;

3. The strong dilatability of the supercritical fluid which plays through the derivative $\left(\partial w_{c}^{e} / \partial T\right)_{P}$ and through the density variation. 
So, the term due to the temperature perturbation results from the coupling between the critical behavior of the solute solubility with respect to temperature and the strong dilatability of the supercritical solvent, and the term due to the pressure perturbation results from the coupling between the critical behavior of the solute solubility with respect to pressure and the strong compressibility of the supercritical solvent, especially the piston effect that it causes. It can be noted that the term due to the density perturbation tends to increase $w$ at the plate $x=0$ and to decrease $w$ at the plate $x=L$. Due to the retrograde phenomenon, the derivative $\left(\partial w_{c}^{e} / \partial T\right)_{P}$, that is very large, is negative. Moreover, it is much larger than $\left(\partial w_{c}^{e} / \partial P\right)_{T}$ (in dimensionless form, $T_{i}\left(\partial w_{c}^{e} / \partial T\right)_{P}\left(T_{i}, P_{i}\right)=-4.96$ whereas $\left.P_{i}\left(\partial w_{c}^{e} / \partial P\right)_{T}\left(T_{i}, P_{i}\right)=0.175\right)$. As a consequence, the term due the temperature perturbation is the largest one in Eq. (13). Thus, the temperature increase of the plate $x=0$ leads to a strong decrease of $w(x=0, t)$ as it is shown in Fig. 6. At the same time, the piston effect, generated by the boundary heating, provokes a fast, strong and homogeneous increase of the pressure in the whole cell (Fig. 7), leading to a strong increase of the term due to the pressure perturbation in Eq. (13). Since the increase of the plate temperature is done using a cosine law, this temperature increase is weaker near the end of the heating phase (i.e. for $t$ approaching $t_{\text {heat }}$ ) and the terms due to the pressure and density perturbations become then the largest ones so that $w(x=0, t)$ begins to increase again before the end of the heating phase $\left(t=t_{\text {heat }}\right)$ (Fig. 6). From $t \approx 10 \mathrm{~s}, w(x=0, t)$ slowly keeps on increasing towards an asymptotic value. The maximum perturbation of $w$ at the heated plate, $\delta w(x=0, t)=w(x=0, t)-w_{i}$, which is obtained about $0.05 \mathrm{~s}$ before the end of the heating phase, is equal to $-7.6973 \times 10^{-5}$, i.e. it corresponds to a decrease about $1 \%$ of the mass fraction of Naphthalene for an increase of $0.0032 \%$ of the plate temperature. The perturbation of the mass fraction of 
Naphthalene is therefore 312 times larger than that of temperature. Since the diffusion coefficient of Naphthalene in supercritical $\mathrm{CO}_{2}$ is very small, the strong decrease of the mass fraction at the plate $x=0$ due to the heating induces a thin diffusion boundary layer that grows very slowly, at the mass diffusion speed (see Fig. 8). To summarize, the leading mechanism governing the evolution of the mass fraction of Naphthalene at the heated plate is the critical behavior of the solubility with respect to temperature. We have just induced a change of the mass fraction of Naphthalene at the plate $x=0$ by modifying the parameters of the interface reaction (i.e. by increasing the plate temperature), exactly as in the case of a perfect gas mixture. However, the response of the system is very different from that of a perfect gas because of the retrograde solubility phenomenon that is specific to supercritical fluids (Raspo et al., 2005). Moreover, the perturbation of the mass fraction resulting from the temperature perturbation is much larger than that obtained in a perfect gas mixture.

On the other hand, the piston effect, coupled with the critical behavior of the solute solubility with respect to pressure, is actually the leading mechanism governing the evolution of the mass fraction of Naphthalene at the isothermal plate $x=L$. Indeed, the term due to the temperature perturbation disappears from Eq. (13) since temperature remains unchanged. The piston effect induced by the heating of the opposite plate provokes a strong and homogeneous increase of the pressure in the whole cell, and therefore at the isothermal interface. Thus, due to the critical behavior of the solubility with respect to pressure, this strong increase of pressure induces a fast and strong increase of $w$ at the plate $x=L$ through the term due to the pressure perturbation in Eq. (13) (Fig. 6). At the end of the heating phase $\left(t=t_{\text {heat }}\right)$, the perturbation of $w$ at the isothermal plate, $\delta w(x=L, t)=w(x=L, t)-w_{i}$, is equal to $5 \times 10^{-5}$. Therefore, it corresponds to an increase about $0.65 \%$ of the mass fraction of Naphthalene, i.e. a 
perturbation 203 times larger than that of temperature. So, we induced a change of the mass fraction at the isothermal plate $x=L$ by modifying the parameters of the interface reaction (namely the pressure) but this modification is generated by a perturbation that is applied at the opposite side of the system and that affects the plate $x=L$ thanks to the non-local character of the piston effect. Therefore, the phenomenon occurring at the isothermal plate is specific to supercritical fluids. The behavior of the system is completely different from that of a perfect gas mixture (Raspo et al., 2005). As at the heated plate, the increase of $w(x=L, t)$ induces, since the beginning of the simulation, a thin diffusion layer which grows at the mass diffusion speed (Fig. 8). There is a very fast mass transfer at the isothermal plate, as at the heated plate, which is illustrated by the evolution of the mass flux $\Phi=\rho D_{21} \partial w / \partial n$ (with $n$ the outward normal to the boundary) at the two reactive interfaces in Fig. 9. Indeed, the mass flux exhibits a very large peak at the end of the heating phase $\left(t=t_{\text {heat }}\right)$. On the other hand, the mass transfer from the plate towards the bulk of the fluid is very slow due to the very small value of the binary diffusion coefficient of Naphthalene in supercritical $\mathrm{CO}_{2}$.

To summarize, thanks to the piston effect (affecting very rapidly the whole system since its characteristic time is less than $0.1 \mathrm{~s}$ ), the heating of the plate $x=0$ causes the fast release of the heterogeneous reaction at the opposite plate, kept at the initial temperature, because of the critical behavior of the solute solubility. The behavior of the interface reactions at the two plates results, from the very beginning of the heating phase, in a mass fraction field divided into three zones just like the temperature field: two thin diffusion boundary layers near the solid plates and the bulk of the cell where the mass fraction of Naphthalene remains unchanged (Fig. 8). After the heating phase (i.e. for $t>t_{\text {heat }}$ ), the mass fraction of Naphthalene at the reactive plates slightly evolves and Naphthalene slowly diffuses in the bulk of the cell. 


\section{2- Proximity of the LCEP}

The above-described behavior of the mass fraction of Naphthalene at the two reactive interfaces was observed for all the considered initial temperatures $T_{i}$ and temperature increases $\Delta T$. However, the proximity of the LCEP influences the value of the mass fraction at the reactive interfaces, as the maximum of the mass flux, since the derivatives of the solubility become smaller and the piston effect becomes less effective as one moves away from the LCEP. Distances to the LCEP ranging from $0.1 \mathrm{~K}$ to $15.5 \mathrm{~K}$ were considered. For $T_{i}=307.75 \mathrm{~K}, 308.15 \mathrm{~K}$ and $309.15 \mathrm{~K}$, the heating phase lasts about $1 \mathrm{~s}$ (more precisely $120 t_{P E}, 10 t_{P E}$ and $2 t_{P E}$ respectively). On the other hand, for $T_{i}=311 \mathrm{~K}$, $318.15 \mathrm{~K}$ and $323.15 \mathrm{~K}$, the characteristic time of the piston effect is larger than $1 \mathrm{~s}$ (see table 2) and the plate temperature is gradually increased during $1 t_{P E}$, since the behavior of the reaction at the isothermal plate is governed by the piston effect. Figure 10 shows the ratio between the relative perturbation of the mass fraction at the two reactive interfaces (i.e. $|\delta w| / w_{i}$ with $\delta w=w(x=0$ or $\left.L, t)-w_{i}\right)$ at $t=t_{\text {heat }}$ and that of the temperature at the heated plate (i.e. $\Delta T / T_{i}$ ) as a function of the dimensionless distance to the LCEP, $\varepsilon$, for $\Delta T=5 \mathrm{mK}$. We noted that, for a given value of $\varepsilon$, this ratio little changes with $\Delta T$; its variation from the value obtained for $\Delta T=5 \mathrm{mK}$ does not exceed $0.2 \%$ for the smallest $\varepsilon$. Very close to the LCEP (for $T_{i}=307.75 \mathrm{~K}$, i.e. $\varepsilon=3.25 \times 10^{-4}$ ), the perturbation of the mass fraction at the heated plate and that at the isothermal interface are respectively about 400 and 300 times larger than the temperature one which causes it. Figure 10 also shows that the ratio $\left(|\delta w| / w_{i}\right) /\left(\Delta T / T_{i}\right)$ at the heated plate is larger than that at the isothermal interface for $\varepsilon<3.41 \times 10^{-2}$, but this tendency is 
inverted for larger values of $\varepsilon$. This behavior change is due to the ratio between the derivative of the solubility with respect to temperature and that with respect to pressure. Indeed, if the contribution of the term due to the density perturbation is neglected, we can write:

$$
\begin{aligned}
& \left|\frac{\delta w(x=0, t)}{w_{i}}\right|>\frac{\delta w(x=L, t)}{w_{i}} \\
& \Leftrightarrow\left[-T_{i}\left(\frac{\partial w_{c}^{e}}{\partial T}\right)_{P}\left(T_{i}, P_{i}\right)\right] /\left[P_{i}\left(\frac{\partial w_{c}^{e}}{\partial P}\right)_{T}\left(T_{i}, P_{i}\right)\right]>2\left(\frac{\delta P(t)}{P_{i}}\right) /\left(\frac{\Delta T}{T_{i}}\right)
\end{aligned}
$$

For $\varepsilon \leq 4.88 \times 10^{-3}$, the ratio between the relative perturbation of pressure and that of temperature is about 3 , and the ratio of the derivatives of $w_{c}^{e}$, little different from that of the derivatives of the solubility, is larger that 6 so that the relation (16) is satisfied. For $\varepsilon \geq 1.09 \times 10^{-2}$, the ratio between the relative perturbation of pressure and that of temperature is about 2, and, for $\varepsilon=5.04 \times 10^{-2}$, the ratio of the derivatives of $w_{c}^{e}$ is smaller than 4 so that the relation (16) is no longer satisfied and, consequently, the perturbation of the mass fraction becomes larger at the isothermal interface than that at the heated plate. Thus, far enough from the LCEP, the heating of the plate at $x=0$ induces a quantity of matter involved in the reaction which is larger at the opposite isothermal plate than that at the heated plate, and the relative perturbation of the mass fraction at $x=L$ nevertheless remains about 20 times larger than that of temperature due to the heating.

We noticed that the ratio $\left(\delta w / w_{i}\right) /\left(\Delta T / T_{i}\right)$ at the isothermal plate behaves like $\varepsilon^{-0.72}$ for $\varepsilon \geq 4.88 \times 10^{-3}$. However, as the LCEP is approached, this law is no longer true and, for $\varepsilon \leq 1.62 \times 10^{-3}$, this ratio behaves only like $\varepsilon^{-0.22}$. Solely an asymptotic 
analysis, for $\varepsilon \rightarrow 0$, could confirm this behavior very close to the LCEP. On the other

hand, the ratio $\left(|\delta w| / w_{i}\right) /\left(\Delta T / T_{i}\right)$ at the heated plate does not evolve as the inverse of a power of $\varepsilon$.

Finally, figure 11 shows the maximum of the mass flux at the two reactive interfaces as a function of the dimensionless distance to the LCEP, $\varepsilon$, for $\Delta T=5 \mathrm{mK}$. As for the relative perturbation of the mass fraction, the mass flux at the heated plate is larger than that at the isothermal interface for $\varepsilon \leq 3.41 \times 10^{-2}$, and the tendency is inverted for larger values of $\varepsilon$.

\section{Conclusion}

Therefore, we have shown that, by heating a supercritical dilute mixture at one side, the piston effect (through the strong and homogeneous increase of pressure that it provokes in the whole fluid) causes the release of a heterogeneous reaction at the opposite plate which was kept at the initial temperature. This phenomenon is possible because of the non-local character of the piston effect and because of the critical behavior of the solubility of a solid in a supercritical fluid with respect to pressure. Simulations in the case of a cooling of the plate $x=0$ were also carried out and a similar phenomenon was observed: the strong decrease of the pressure caused by the piston effect induces a fast and strong decrease of the mass fraction at the isothermal plate, leading to similar but reversed profiles of the mass fraction (Fig. 12). The route is now opened towards the exploration of the coupling between convection and heterogeneous reactions in supercritical mixtures, including hydrothermal synthesis and crystal growth. 


\section{Notation}

a

b covolume in the Peng-Robinson equation.

$\mathrm{D}_{21} \quad$ diffusion coefficient.

$\mathrm{k}_{12}, \mathrm{l}_{12} \quad$ binary interaction parameters in the mixing rules.

L distance between the two reactive plates.

M molecular weight.

P pressure.

$\delta \mathrm{P} \quad$ perturbation of the pressure.

Pr Prandtl number.

$\mathrm{P}_{\mathrm{th}} \quad$ thermodynamic pressure in the low Mach number approximation.

$\mathrm{p} \quad$ dynamic pressure in the low Mach number approximation.

$\mathrm{R} \quad$ perfect gas constant.

t time.

$t_{d} \quad$ characteristic time of thermal diffusion.

$t_{\mathrm{Md}} \quad$ characteristic time of mass diffusion.

$t_{\mathrm{PE}} \quad$ characteristic time of the piston effect.

T temperature.

$\Delta \mathrm{T} \quad$ temperature increase applied at the plate $\mathrm{x}=0$.

u velocity.

$\mathrm{V} \quad$ molar volume.

w mass fraction of Naphthalene.

$\delta \mathrm{w} \quad$ perturbation of the mass fraction at the reactive plates. space coordinate. 
$\mathrm{y}_{2} \quad$ mole fraction of Naphthalene.

\section{Greek letters}

$\alpha \quad$ thermal diffusivity.

$\beta \quad$ thermal expansion coefficient.

$\chi \quad$ isothermal compressibility.

$\varepsilon \quad$ dimensionless distance to the gas-liquid critical point for the pure compound or to the Lower Critical EndPoint (LCEP) for the mixture.

$\phi \quad$ association factor.

$\Phi \quad$ mass flux at the solid interfaces.

$\eta \quad$ viscosity.

$\varphi \quad$ fugacity coefficient.

$\rho$ density.

$\omega$ acentric factor.

\section{Subscript}

b value at boiling point.

c value at the gas-liquid critical point.

i value at the initial state.

$\mathrm{j} \quad$ refers to component $\mathrm{j}$ of the mixture.

LCEP value at the LCEP.

1 refers to $\mathrm{CO}_{2}$.

2 refers to Naphthalene. 


\section{Superscript}

cal calculated value.

e value for solid-supercritical fluid equilibrium.

exp experimental value.

s value in the solid phase.

sub value at sublimation.

\section{Acknowledgements}

The authors acknowledge the financial support from the CNES (Centre National d'Etudes Spatiales). They gratefully thank Pr. Evelyne Neau and Dr. Christophe Nicolas (MSNM-GP) and Dr. Roger Prud'homme (LMM, UMR 7607, Paris, France) for their friendly help and fruitful discussions.

\section{Appendix A}

Coefficients $\Lambda$ and $\Gamma$ appearing in Eq. (4) are defined by:

$$
\begin{aligned}
& \Lambda=\left[\frac{A_{1}}{M_{1}}+\frac{B_{1}}{M_{1}} \frac{T}{2}+\frac{C_{1}}{M_{1}} \frac{T^{2}}{3}+\frac{D_{1}}{M_{1}} \frac{T^{3}}{4}+\frac{E_{1}}{M_{1}} \frac{T^{4}}{5}\right]+w \Omega(T) \\
& +\frac{\theta(w)}{2 \sqrt{2} b(w)}\left[\frac{a(T, w)}{T}-\left(\frac{\partial a}{\partial T}\right)_{w}\right] \operatorname{Ln}\left(\frac{1+(1-\sqrt{2})(b(w) / \theta(w)) \rho}{1+(1+\sqrt{2})(b(w) / \theta(w)) \rho}\right) \\
& \Gamma=\Omega\left(T_{0}\right) T_{0}+\frac{H_{2}\left(T_{0}\right)}{M_{2}}-\frac{H_{1}\left(T_{0}\right)}{M_{1}}
\end{aligned}
$$

with $T_{0}=298.15 \mathrm{~K}, H_{2}\left(T_{0}\right)$ and $H_{1}\left(T_{0}\right)$ the perfect gas enthalpy of Naphthalene and $\mathrm{CO}_{2}$ respectively at temperature $T_{0}$, and: 


$$
\Omega(T)=\left(\frac{A_{2}}{M_{2}}-\frac{A_{1}}{M_{1}}\right)+\left(\frac{B_{2}}{M_{2}}-\frac{B_{1}}{M_{1}}\right) \frac{T}{2}+\left(\frac{C_{2}}{M_{2}}-\frac{C_{1}}{M_{1}}\right) \frac{T^{2}}{3}+\left(\frac{D_{2}}{M_{2}}-\frac{D_{1}}{M_{1}}\right) \frac{T^{3}}{4}+\left(\frac{E_{2}}{M_{2}}-\frac{E_{1}}{M_{1}}\right) \frac{T^{4}}{5}
$$

where the coefficients $A_{j}, B_{j}, C_{j}, D_{j}, E_{j}$ come from the expression of the heat capacity at constant pressure for the perfect gas (Poling et al., 2001):

$$
C_{p j}(T)=A_{j}+B_{j} T+C_{j} T^{2}+D_{j} T^{3}+E_{j} T^{4}
$$

\section{Appendix B}

The thermal diffusivity $\alpha$ of $\mathrm{CO}_{2}$ at initial conditions is defined by the formula:

$$
\alpha=\frac{\lambda}{\rho_{i} C_{P}}
$$

The specific heat at constant pressure $C p$ is computed from the specific heat at constant volume $C_{V}$, that is fixed at its value for the perfect gas $\left(C_{V}=C_{V_{0}}=648.04 \mathrm{~J} . \mathrm{kg}^{-1} . \mathrm{K}\right)$, using the thermodynamic equation:

$$
C_{P}=C_{V}+\frac{T_{i}}{\rho_{i}^{2}}\left(\frac{\partial P}{\partial T}\right)_{\rho}^{2}\left(\frac{\partial \rho}{\partial P}\right)_{T}
$$

in which the derivatives are calculated using the Peng-Robinson equation for pure $\mathrm{CO}_{2}$.

\section{References}

Afrane, G., Chimowitz, E. H., (1995). Adsorption in near critical binary solvent mixtures: thermodynamic analysis and data. Fluid Phase Equilibria, 111, 213-238. 
Amiroudine, S., Ouazzani, J., Carles, P., Zappoli, B., (1997). Numerical solutions of 1D unsteady near-critical fluid flows using finite volume methods. Eur. J. Mech. B/Fluids, $16,655-680$.

Arai, Y., Sako, T., and Y. Takebayashi, Y., (2002). Supercritical fluids: molecular interactions, physical properties and new applications, Springer.

Boukari, H., Shaumeyer, J. N., Briggs, M. E., Gammon, R. W., (1990). Critical speeding up in pure fluids. Physical Review A, 41, 2260-2263.

Garrabos, Y., Bonetti, M., Beysens, D., Perrot, F., Fröhlich, T., Carles, P., Zappoli, B., (1998). Relaxation of a supercritical fluid after a heat pulse in the absence of gravity effects: theory and experiments. Physical Review E, 57, 5665-5681.

Guenoun, P., Khalil, B., Beysens, D., Garrabos, Y., Kammoun, F., Le Neindre, B., Zappoli, B., (1993). Thermal cycle around the critical point of carbon dioxide under reduced gravity. Physical Review E, 47, 1531-1540.

Higashi, H., Iwai, Y., Takahashi, Y., Uchida, H., Arai, Y., (1998). Diffusion coefficients of naphthalene and dimethylnaphthalene in supercritical carbon dioxide. Fluid Phase Equilibria, 144, 269-278.

Higashi, H., Iwai, Y., Arai, Y., (2001). Solubilities and diffusion coefficients of high boiling compounds in supercritical carbon dioxide. Chemical Engineering Science, 56, 3027-3044.

Jha, S. K., Madras, G., (2004). Modeling of adsorption equilibria in supercritical fluids. Journal of Supercritical Fluids, 32, 161-166.

Kogan, A. B., Meyer, H., (1998). Density response and equilibration in a pure fluid near the liquid-vapor critical point. Journal of Low Temperature Physics, 112, 417-448. 
Nicolas, C., Neau, E., Meradji, S., Raspo, I., (2005). The Sanchez-Lacombe Lattice fluid model for the modeling of solids in supercritical fluids. Fluid Phase Equilibria, 232, 219-229.

Nishiumi, H., Fujita, M., Agou, K., (1996). Diffusion of acetone in supercritical carbon dioxide. Fluid Phase Equilibria, 117, 356-363.

Onuki, A., Hao, H., Ferrell, R., (1990). A. Fast adiabatic equilibration in a singlecomponent fluid near the liquid-vapor critical point. Physical Review A, 41, 2256-2259. Paolucci, S., (1982). On the filtering of sound from the Navier-Stokes equations (SAND report 82-8257).

Poling, B. E., Prausnitz, J. M., O'Connell, J. P., (2001) The properties of gases and liquids, $5^{\text {th }}$ edition. McGraw-Hill International Editions.

Raspo, I., Meradji, S., Zappoli, B., (2005). Fast mass transfer at a solid-supercritical fluid interface by piston effect. Progress in Computational Heat and Mass Transfer, Lavoisier, p. 132-137.

Straub, J., Eicher, L., Haupt, A., (1995). The process of heat and mass transport at the critical point of pure fluids. International Journal of Thermophysics, 16, 1051-1058.

Tapia-Corzo, C., Kumaran, V., Chimowitz, E. H., (2000). Experimental study of retrograde adsorption in supercritical fluids. Journal of Supercritical Fluids, 17, 25-33.

Tsekhanskaya, Y. V., Iomtev, M. B., Mushkina, E. V., (1962). Solubility of diphenylamine and naphthalene in carbon dioxide under pressure. Russian Journal of Physical Chemistry, 36 (10), 1177-1181.

Tsekhanskaya, Y. V., (1971). Diffusion of naphthalene in carbon dioxide near the liquid-gas critical point. Russian Journal of Physical Chemistry, 45, 744. 
Uchida, H., Iwai, Y., Amiya, M., Arai, Y., (1997). Adsorption behaviors of 2,6 and 2,7dimethylnaphthalene in supercritical carbon dioxide using NaY-type zeolite. Industrial and Engineering Chemistry Research, 36, 424-429.

Zappoli, B., Bailly, D., Garrabos, Y., Le Neindre, B., Guenoun, P., Beysens, D., (1990). Anomalous heat transport by the piston effect in supercritical fluids under zero gravity. Physical Review A, 41, 2264-2267.

Zappoli, B., Carles, P., (1996). Speeding up of heterogeneous reactions in near-critical phases. Acta Astronautica, 38 (1), 39-45.

Zhong, F., Kogan, A. B., Meyer, H., (1997). Thermal response of a fluid near its critical point: $3 \mathrm{He}$ at $\mathrm{T}>\mathrm{T}_{\mathrm{c}}$. Journal of Low Temperature Physics, 108, 161-190. 


\section{FIGURES}

Fig. 1. Phase diagram of binary mixtures of interest. (CP: Critical Point; LCEP: Lower Critical End-Point; UCEP: Upper Critical End-Point; S=L: solid-liquid equilibrium curve; $\mathrm{S}_{2} \mathrm{LG}$ : solid-liquid-gas curve; dotted lines: liquid-gas critical lines of the mixture).

Fig. 2. Physical configuration.

Fig. 3. Solubility of Naphthalene in supercritical $\mathrm{CO}_{2}$, predicted by the model, versus pressure for $T=308.15 \mathrm{~K}, T=318.15 \mathrm{~K}$ and $T=323.15 \mathrm{~K}$.

Fig. 4. Profiles of the temperature perturbation at several times for $T_{i}=308.15 \mathrm{~K}$ and $\Delta T=10 \mathrm{mK}$.

Fig. 5. Profiles of the density perturbation at several times for $T_{i}=308.15 \mathrm{~K}$ and $\Delta T=10 \mathrm{mK}$.

Fig. 6. Evolution of the mass fraction of Naphthalene $w$ at the heated plate $x=0$ and at the isothermal plate $x=L$ for $T_{i}=308.15 \mathrm{~K}$ and $\Delta T=10 \mathrm{mK}$. The small figure shows the evolution at the very beginning of the simulation, corresponding to twice the heating time $t_{\text {heat }}$.

Fig. 7. Evolution of the total pressure $P$ for $T_{i}=308.15 \mathrm{~K}$ and $\Delta T=10 \mathrm{mK}$.

Fig. 8. Profiles of the perturbation of the mass fraction at several times for $T_{i}=308.15 \mathrm{~K}$ and $\Delta T=10 \mathrm{mK}$.

Fig. 9. Evolution of the mass flux at the heated plate $x=0$ and at the isothermal plate $x=L$ for $T_{i}=308.15 \mathrm{~K}$ and $\Delta T=10 \mathrm{mK}$.

Fig. 10. Ratio of the relative perturbation of the mass fraction of Naphthalene at the two plates, $\mid w\left(x=0\right.$ or $\left.L, t_{\text {heat }}\right)-w_{i} \mid / w_{i}$, at the end of the heating phase and that of the temperature, $\Delta T / T_{i}$, versus the dimensionless distance to the LCEP, $\varepsilon$, for $\Delta T=5 \mathrm{mK}$. 
Published in Chemical Engineering Science, 62, pp. 4182-4192, 2007

Fig. 11. Maximum of the mass flux at the two plates versus the dimensionless distance to the LCEP, $\varepsilon$, for $\Delta T=5 \mathrm{mK}$.

Fig. 12. Profiles of the perturbation of the mass fraction at several times for $T_{i}=308.15 \mathrm{~K}$ in the case of a cooling of the plate $x=0$ for $\Delta T=-10 \mathrm{mK}$. 
Published in Chemical Engineering Science, 62, pp. 4182-4192, 2007

\section{TABLES}

Table 1: Pure component properties.

Table 2: Initial states $\left(T_{i}, P_{i}, w_{i}\right)$ considered in the study and the corresponding values of the dimensionless distance to the LCEP $\varepsilon$, of the thermal diffusivity $\alpha$, of the diffusion coefficient $D_{21}$ and of the various characteristic times (thermal diffusion $t_{d}$, piston effect $t_{P E}$, mass diffusion $\left.t_{M d}\right)$. 


\begin{tabular}{lcccccc}
\hline & $\begin{array}{c}T_{c} \\
(K)\end{array}$ & $\begin{array}{c}\rho_{c} \\
\left(\mathrm{~kg} . \mathrm{m}^{-3}\right)\end{array}$ & $\begin{array}{c}M \\
\left(\mathrm{~kg} \mathrm{~mol}^{-1}\right)\end{array}$ & $\omega$ & $v^{s}$ & $v_{b}$ \\
& 304.21 & 467.8 & $4.401 \times 10^{-2}$ & 0.225 & - & - \\
$\mathrm{CO}_{2}(1)$ & 748.40 & 314.9 & $1.282 \times 10^{-1}$ & 0.302 & $1.1 \times 10^{-4}$ & 155 \\
Naphthalene (2) & $\left.7 \mathrm{~cm}^{3} \cdot \mathrm{mol}^{-1}\right)$ \\
\hline
\end{tabular}

Table 1. 


\begin{tabular}{ccccccccc}
\hline$T_{i}(\mathrm{~K})$ & $\varepsilon$ & $P_{i}(\mathrm{MPa})$ & $w_{i}$ & $\alpha\left(\mathrm{m}^{2} \cdot \mathrm{s}^{-1}\right)$ & $D_{21}\left(\mathrm{~m}^{2} \cdot \mathrm{s}^{-1}\right)$ & $t_{d}(\mathrm{~s})$ & $t_{M d}(s)$ & $t_{P E}(s)$ \\
\hline 307.75 & $3.25 \times 10^{-4}$ & 7.836815 & $7.5216 \times 10^{-3}$ & $8.8941 \times 10^{-9}$ & $2.2298 \times 10^{-8}$ & 11243 & 4485 & 0.0084 \\
308.15 & $1.62 \times 10^{-3}$ & 7.909092 & $7.6751 \times 10^{-3}$ & $9.6465 \times 10^{-9}$ & $2.2327 \times 10^{-8}$ & 10366 & 4479 & 0.094 \\
309.15 & $4.88 \times 10^{-3}$ & 8.089188 & $8.0713 \times 10^{-3}$ & $1.1375 \times 10^{-8}$ & $2.2399 \times 10^{-8}$ & 8791 & 4464 & 0.49 \\
311 & $1.09 \times 10^{-2}$ & 8.420040 & $8.8531 \times 10^{-3}$ & $1.4112 \times 10^{-8}$ & $2.2533 \times 10^{-8}$ & 7086 & 4438 & 1.62 \\
318.15 & $3.41 \times 10^{-2}$ & 9.666847 & $1.2557 \times 10^{-2}$ & $2.1632 \times 10^{-8}$ & $2.3051 \times 10^{-8}$ & 4623 & 4338 & 9.02 \\
323.15 & $5.04 \times 10^{-2}$ & 10.503144 & $1.5929 \times 10^{-2}$ & $2.5724 \times 10^{-8}$ & $2.3413 \times 10^{-8}$ & 3887 & 4271 & 16.2 \\
\hline
\end{tabular}

Table 2. 


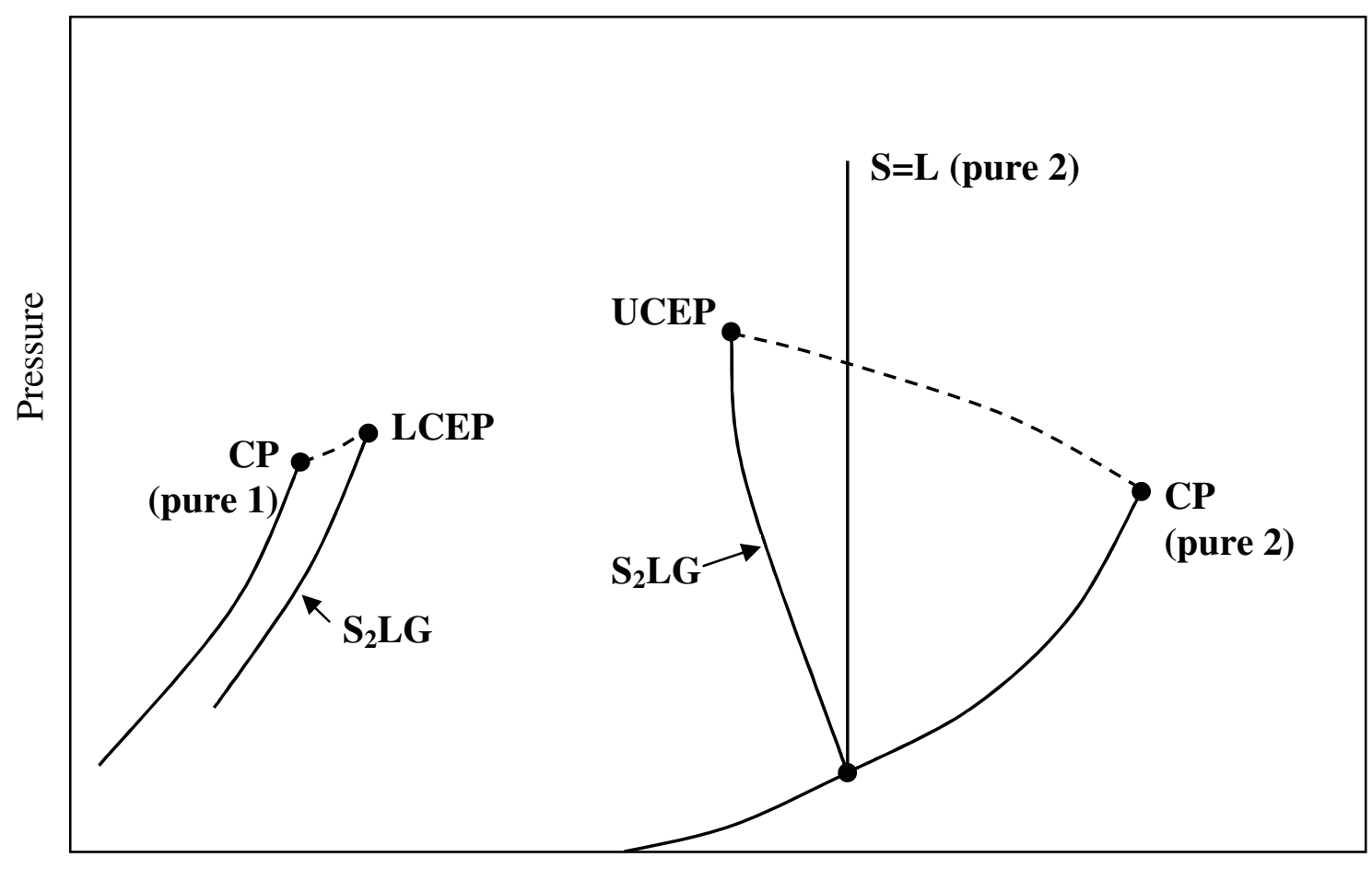

Temperature

Fig. 1 


\begin{tabular}{|c|c|c|}
\hline $\begin{array}{l}\text { Active solid plate } \\
\text { Pure Naphthalene) }\end{array}$ & Dilute mixture & $\begin{array}{l}\text { Active solid plate } \\
\text { (Pure Naphthalene) }\end{array}$ \\
\hline$T_{i}+\Delta T$ & $\mathrm{CO}_{2}+$ Naphthalene & $T_{i}=(1+\varepsilon) T_{L C E P}$ \\
\hline & & $10^{-2} \mathrm{~m}$ \\
\hline
\end{tabular}

Fig. 2 


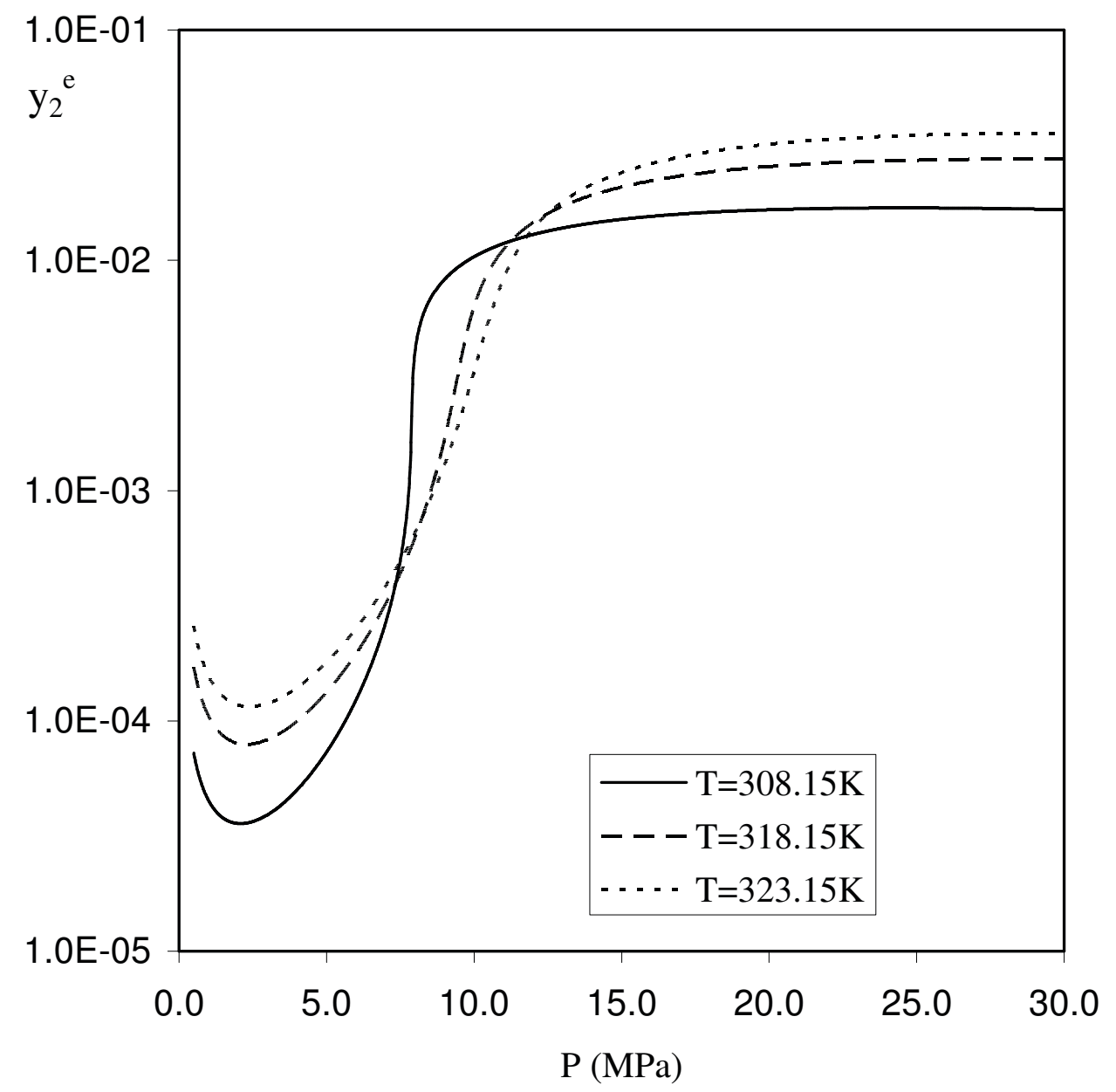

Fig. 3 


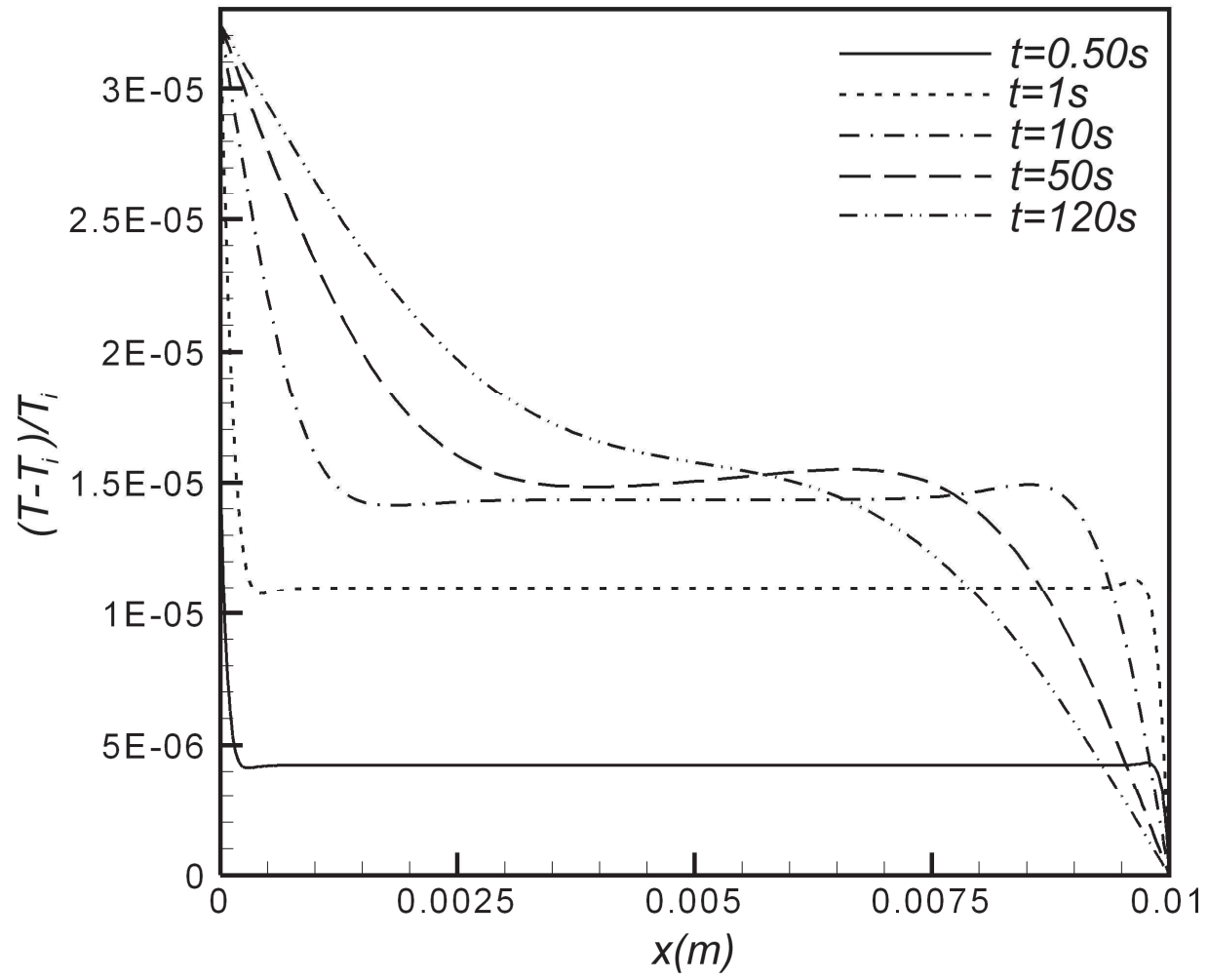

Fig. 4 


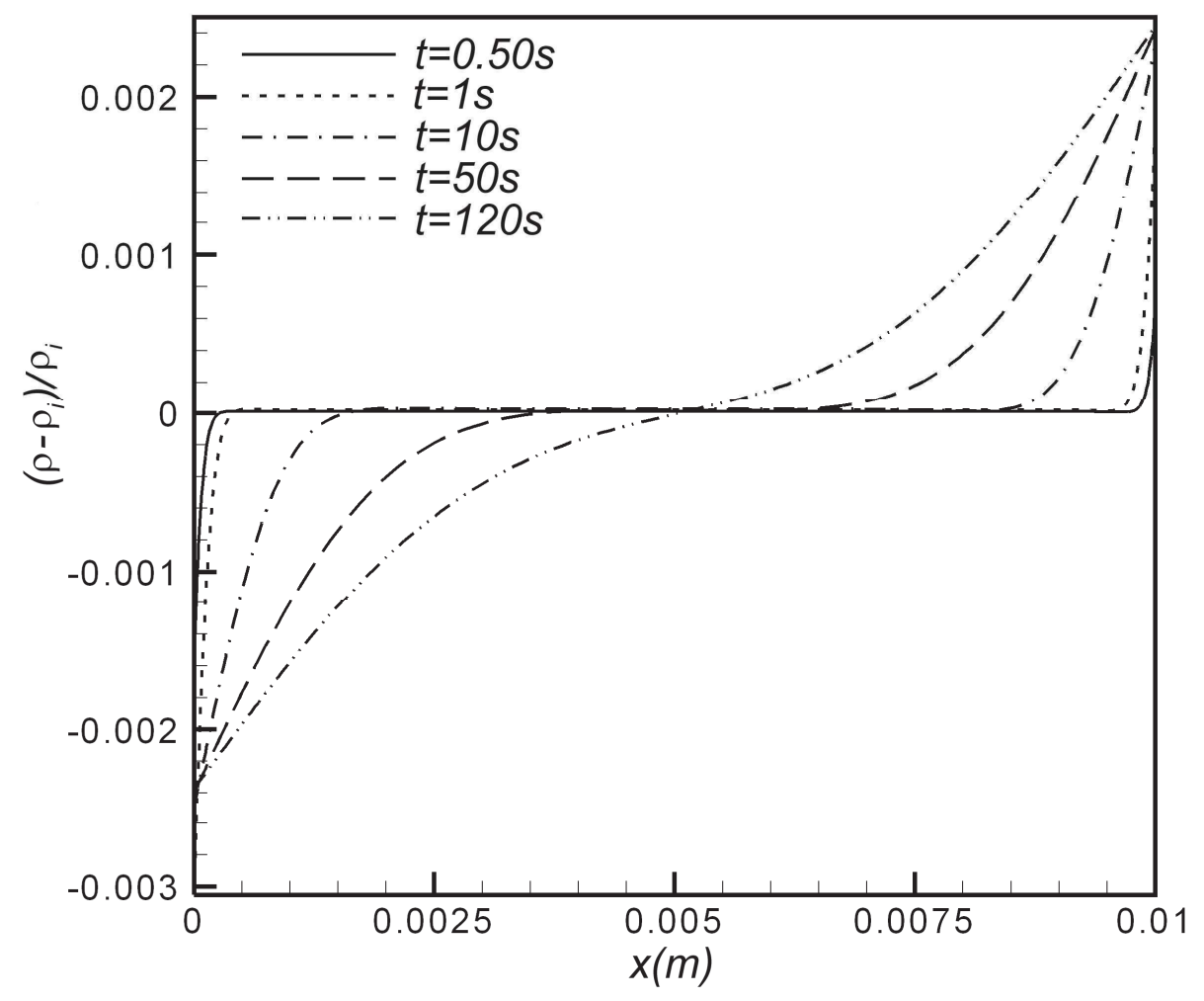

Fig. 5 


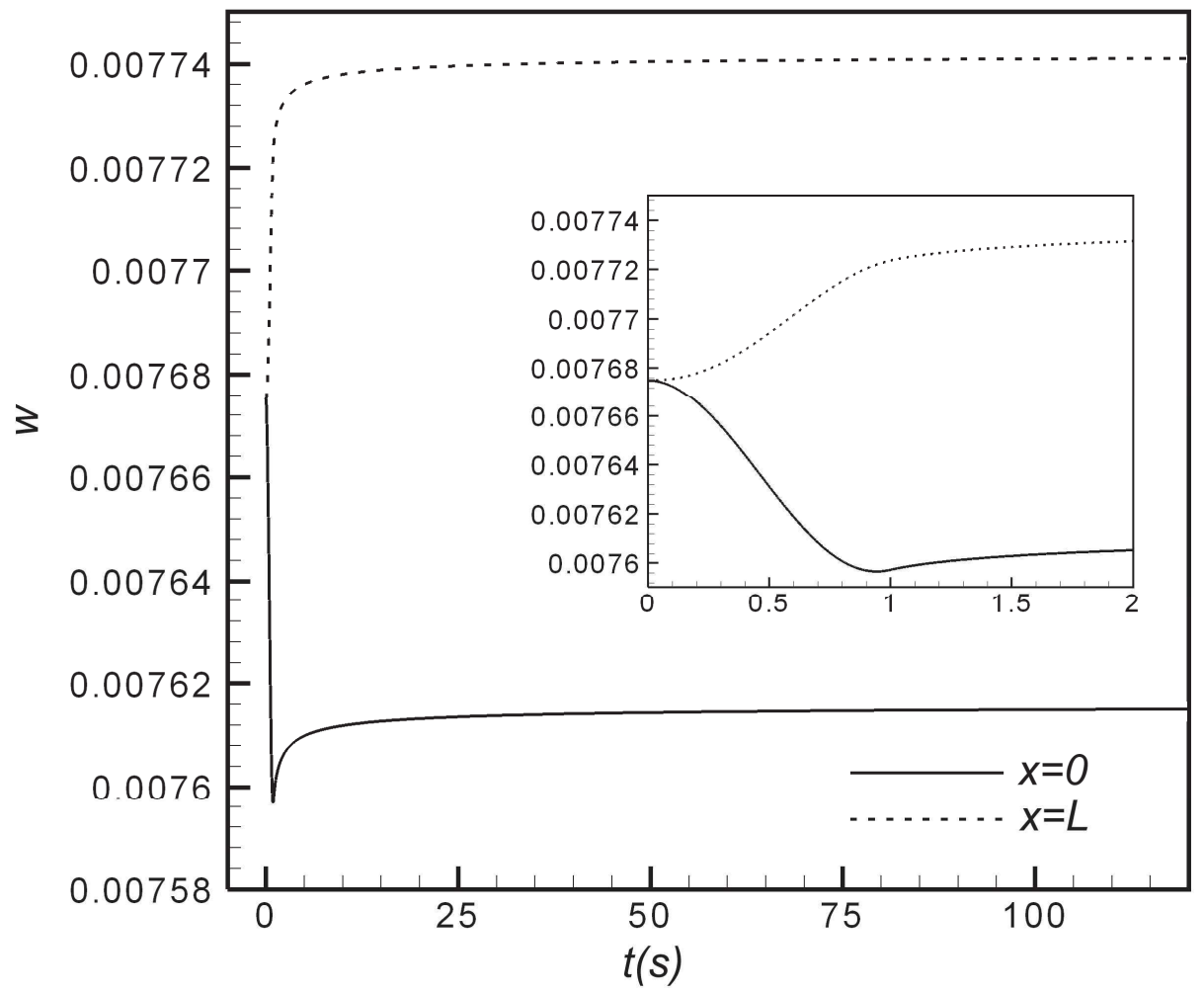

Fig. 6 


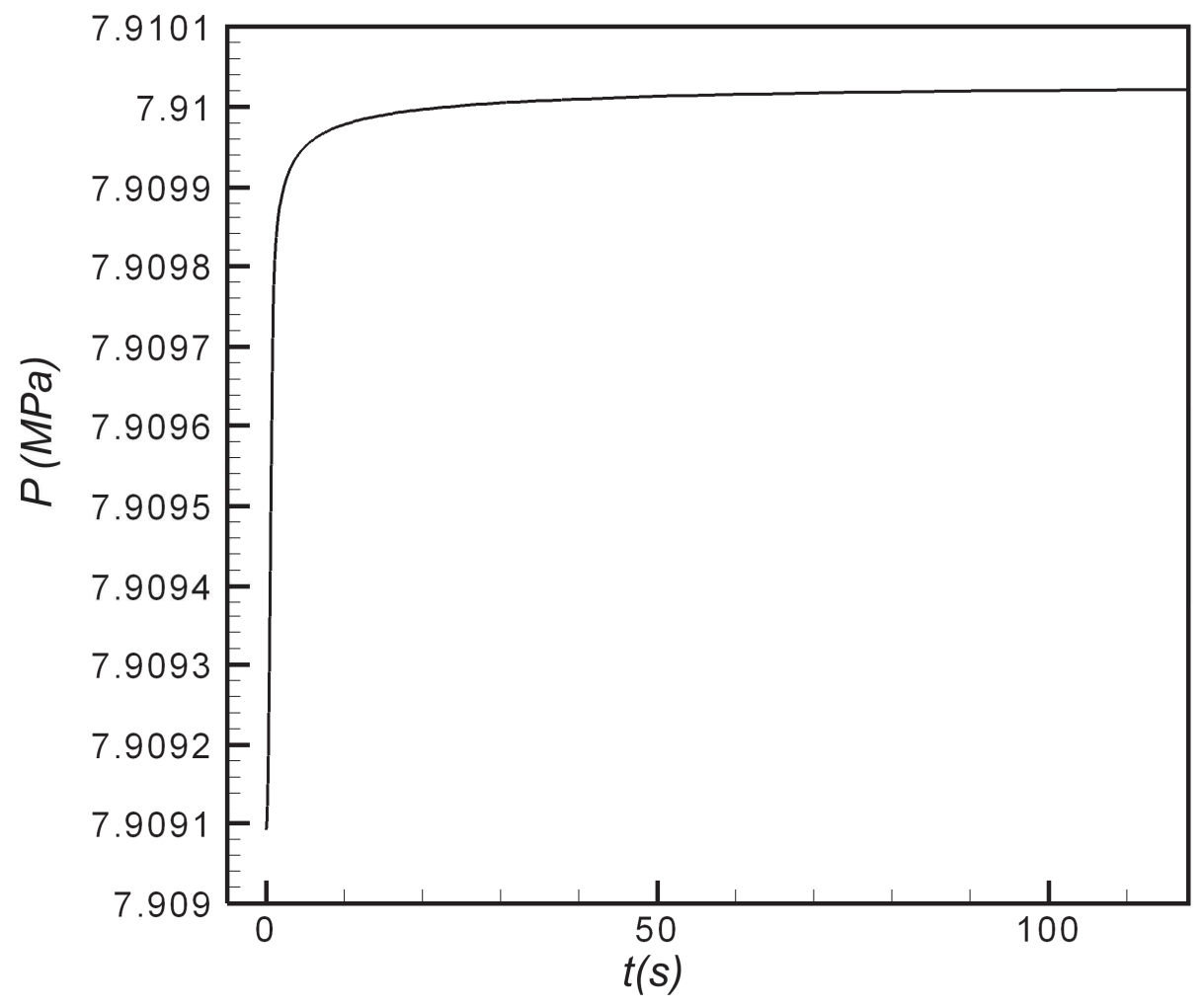

Fig. 7 


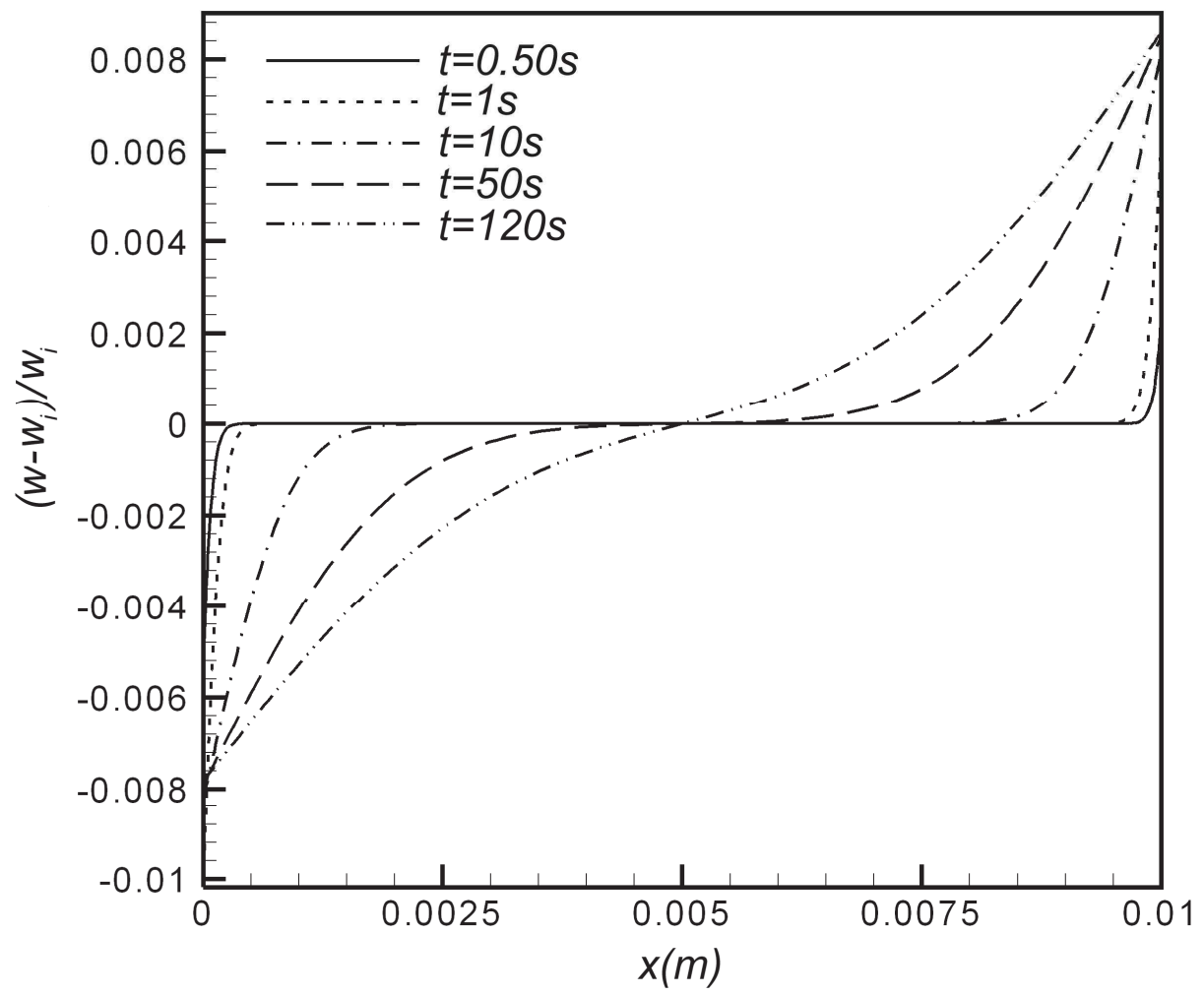

Fig. 8 


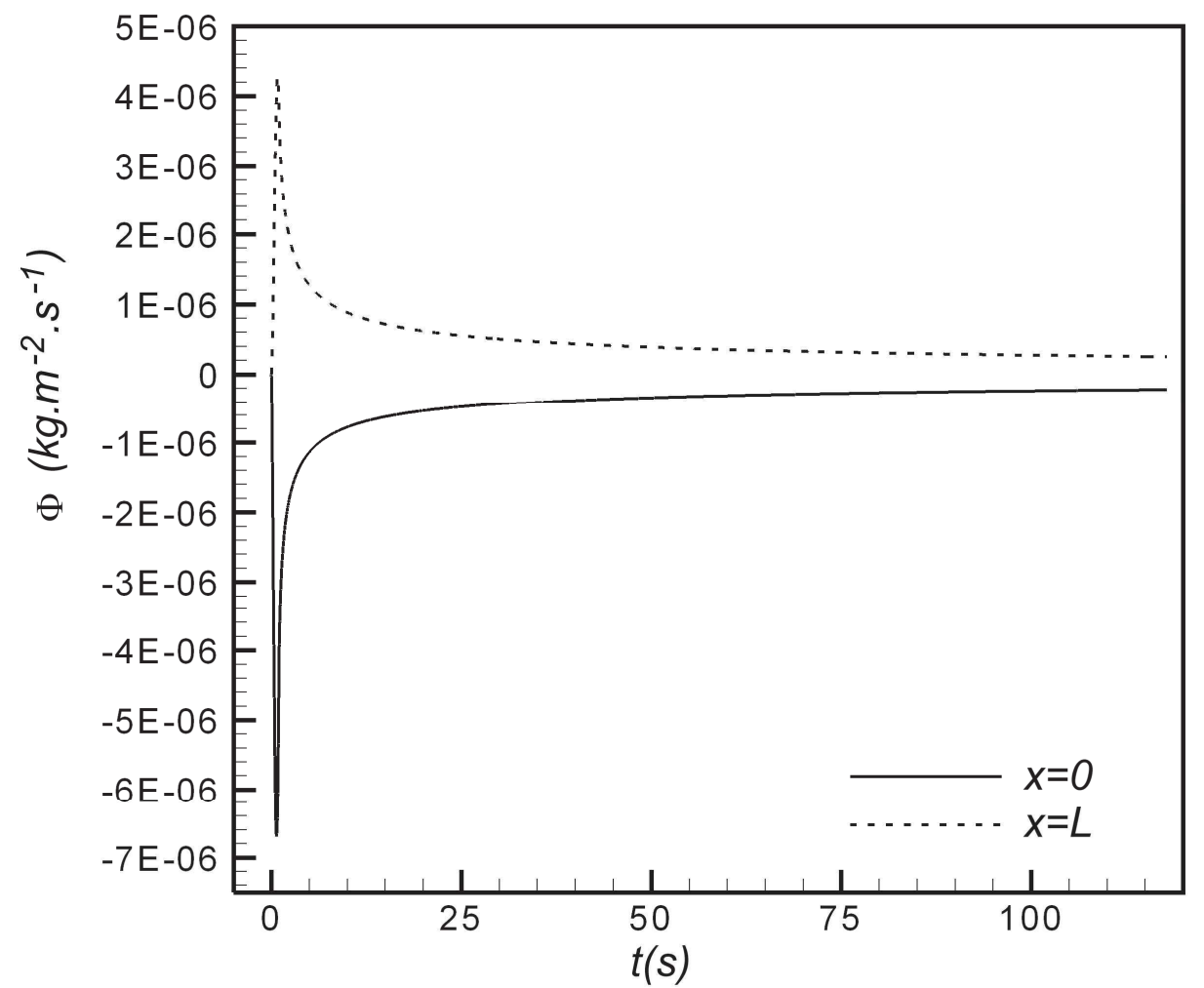

Fig. 9 


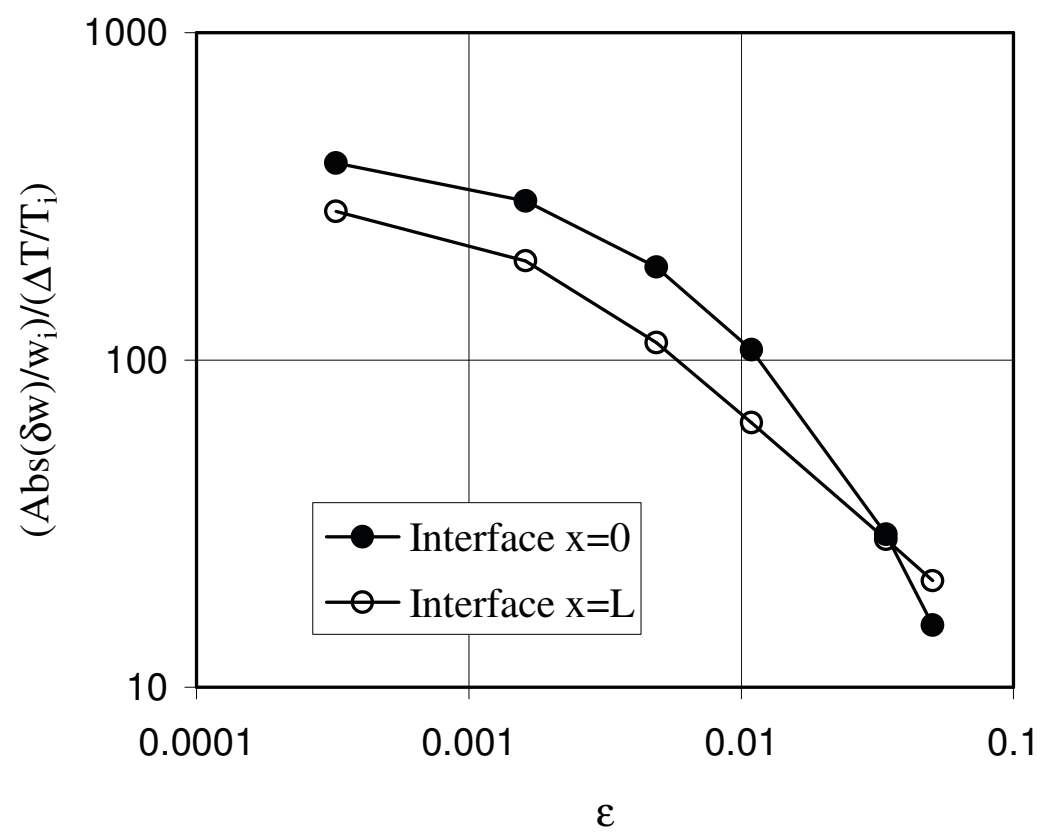

Fig. 10 


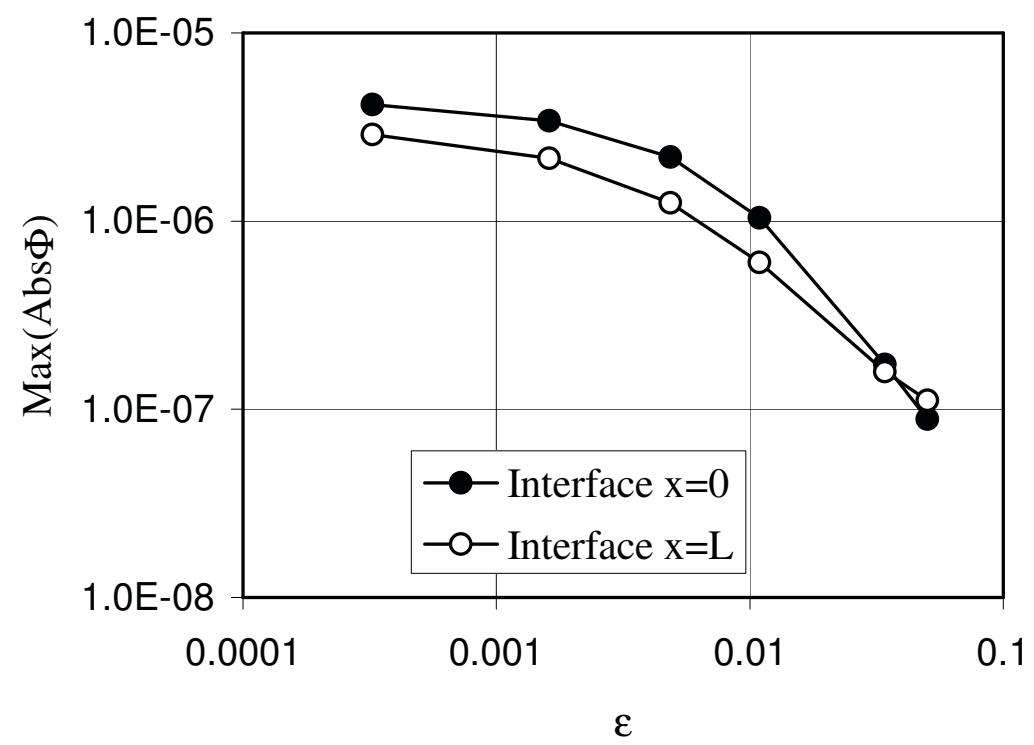

Fig. 11 


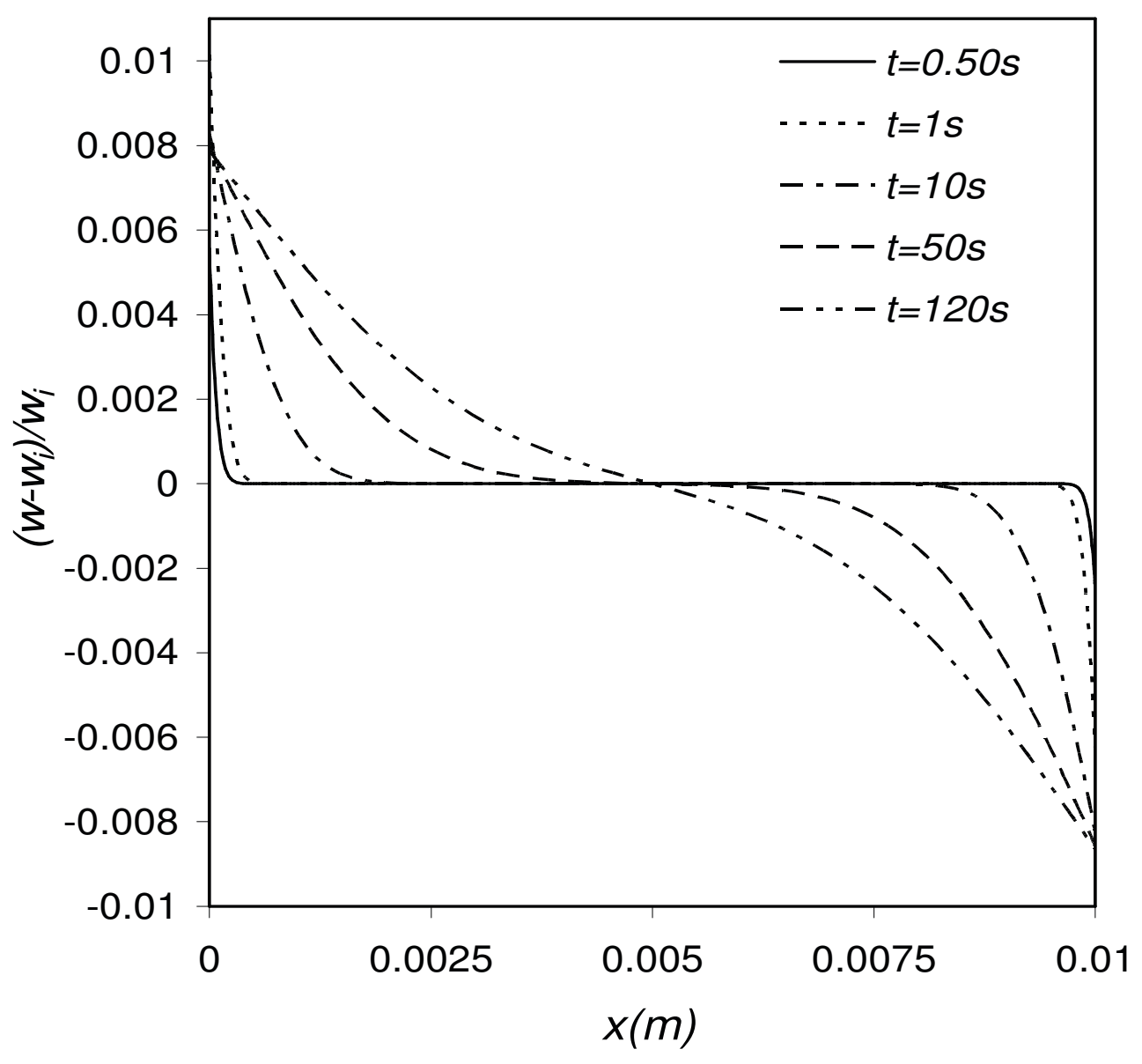

Fig. 12 